\title{
Stiffness gradient in the crystalline lens
}

\author{
Henk A. Weeber • Gabriele Eckert • \\ Wolfgang Pechhold • Rob G. L. van der Heijde
}

Received: 28 August 2006 / Revised: 10 January 2007 / Accepted: 14 January 2007 / Published online: 7 February 2007

(C) Springer-Verlag 2007

\begin{abstract}
Background While the overall stiffness of the lens has been measured in a number of studies, the knowledge about the stiffness distribution within the lens is still limited. The purpose of this study was to determine the stiffness gradient in the human crystalline lens. A secondary purpose was to determine whether the stiffness gradient depends on age.

Methods The local dynamic stiffness was measured in 10 human crystalline lenses (age range: 19 to 78 years). The lenses were stored at $-70^{\circ} \mathrm{C}$ before being measured. The influence of freezing on the mechanical properties has been determined in a previous study. A small oscillating probe was used to measure the local dynamic shear modulus as a measure of lens stiffness. The measurements were taken in the cross-sectional plane through the lens equator.

Results The local dynamic shear modulus varied with location for all tested lenses. The central stiffness of the
\end{abstract}

H. A. Weeber $(\bowtie)$

AMO,

Van Swietenlaan 5,

9728 NX Groningen, The Netherlands

e-mail: henk.weeber@amo-inc.com

G. Eckert

Carl Zeiss SMT AG,

Carl Zeiss Strasse 22,

73446 Oberkochen, Germany

W. Pechhold

Institute of Dynamic Materials Testing, University of Ulm,

Helmholtzstrasse 20,

89069 Ulm, Germany

R. G. L. van der Heijde

VU Medical Centre,

P.O. Box 7057, 1007 MB Amsterdam, The Netherlands oldest lens ( 78 years) was $10^{4}$ times higher than the youngest (19 years) lens. The equatorial stiffness of the oldest lens was $10^{2}$ times higher than the youngest lens. For the older lenses, the centre was 5.8-210 times stiffer than the periphery, as opposed to earlier results described by Fisher (1971), who found that the periphery was up to 3 times softer than the centre for lenses younger than 70-years-old. For the three youngest lenses (19 to 49 years), the periphery was 2.2-16.6 times stiffer than the centre.

Conclusions The dynamic stiffness of the crystalline lens varies with location within the lens. The stiffness gradient depends on the age of the lens. The results of the 10 lenses indicate that the stiffness of both centre and periphery increase with age, but at a different rate.

Keywords Lens - Accommodation . Biomechanical properties $\cdot$ Stiffness

\section{Introduction}

The mechanical properties of the crystalline lens influence the ability of the lens to change its shape during accommodation. Consequently, the stiffness of the human crystalline lens is thought to have an effect on the accommodative ability and the development of presbyopia [3, 18-20]. The young and soft crystalline lens is easy to deform, while the old lens is stiff, hard and unable to be deformed [3, 18, 31, 32]. While some authors represent the stiffness of the lens using a single value for the whole lens $[2,24]$, e.g. the Young's modulus, it has been shown that the stiffness of the lens varies, depending on the location within the lens where stiffness is measured [20]. Also measurements of lens hardness $[30,35]$ have shown that there are mechanical differences between the lens nucleus and the cortex. This is 
further supported by lens morphology $[1,36]$, which shows a compaction of fibres toward the lens centre. Other differences have also been observed between the lens nucleus and cortex in terms of the protein gradient [13], refractive index [33] and acoustic parameters [26].

Numerous studies have been conducted to measure crystalline lens stiffness $[2,4,10,12,15,16,19-22,28]$; however, only a few of these studies consider differences between the nucleus and cortex. Several studies have demonstrated that during accommodation, the axial deformation in the lens takes place predominantly in the nucleus $[7,11,23,29]$. This phenomenon seems to contradict the idea of a crystalline lens having a uniform and isotropic stiffness throughout the entire lens.

Fisher [15] determined the stiffness of human lenses using lens-spinning experiments. Nuclear and cortical stiffness were calculated, based on the geometric changes of the lens during spinning. Fisher concluded that the lens nucleus is softer than the cortex at all ages between 0 and 70 years. However, a recently published analysis suggests that this conclusion, particularly in middle age, may be an artefact associated with systematic errors, caused by the modelling assumptions inherent in Fisher's method [9]. Dynamic mechanical analysis (DMA) measurements by Kuwahara [28] on bovine lenses showed that the lens nucleus is indeed stiffer than the lens as a whole. Pau and Kranz [30] measured the force required to penetrate crystalline lenses with a conical probe. For lenses 20 years or older, a higher force was required to achieve axial lens penetration of the nucleus, compared to the cortex. Heys et al. [20] did a similar experiment, in which they measured the penetration force at different locations on a lens cross-section, showing a large difference between the central and peripheral stiffness of human lenses.

The stiffness of a viscoelastic material depends on the deformation rate [14]. Therefore, we used dynamic measurements, using multiple deformation rates. The measurement methods were based on dynamic measurement techniques that were also used in an earlier study on human lenses [39].

The present study aimed to determine the local stiffness of the human crystalline lens and possible changes with age. We show that the lens stiffness depends strongly on the location within the lens where stiffness is measured, and that not only the stiffness, but also the stiffness profile/ gradient, changes dramatically with age.

\section{Methods}

Testing procedure

Ten human lenses ranging in age from 19 to 78 years were obtained from donor eyes from the Cornea Bank in Halle.
The eyes had no known ocular diseases. The eyes were frozen immediately after enucleation and stored at $-70^{\circ} \mathrm{C}$. The sample collection followed the tenets of the Declaration of Helsinki.

The lenses were subjected to dynamic mechanical analysis (DMA). While a lens sample was held in a sample holder, a load (displacement) is applied on one side of the sample and the reaction force is measured at the opposite side. The applied load is a sinusoidal displacement (oscillation), which is defined by its amplitude and frequency. As long as the measurement takes place in the linear viscoelastic range, the measured force is proportional to the amplitude of the applied displacement. The measurement procedure has been described previously [39], when it was used for determining the mechanical properties of the entire lens. In the current setup, a lens half was placed in a sample holder, while the load consisted of a vertical oscillation of a cylindrical probe, which was inserted into the lens at several positions across the cut lens surface (Fig. 1).

In preparation of a measurement, the lens was cut in half along the equatorial plane, while the lens was still in a halffrozen state. The lens half was positioned in a lens-shaped sample holder of a size that fitted the lens. The cut surface of the lens was covered with a thin aluminium plate (thickness $1.0 \mathrm{~mm}$ ), which had small openings (holes with

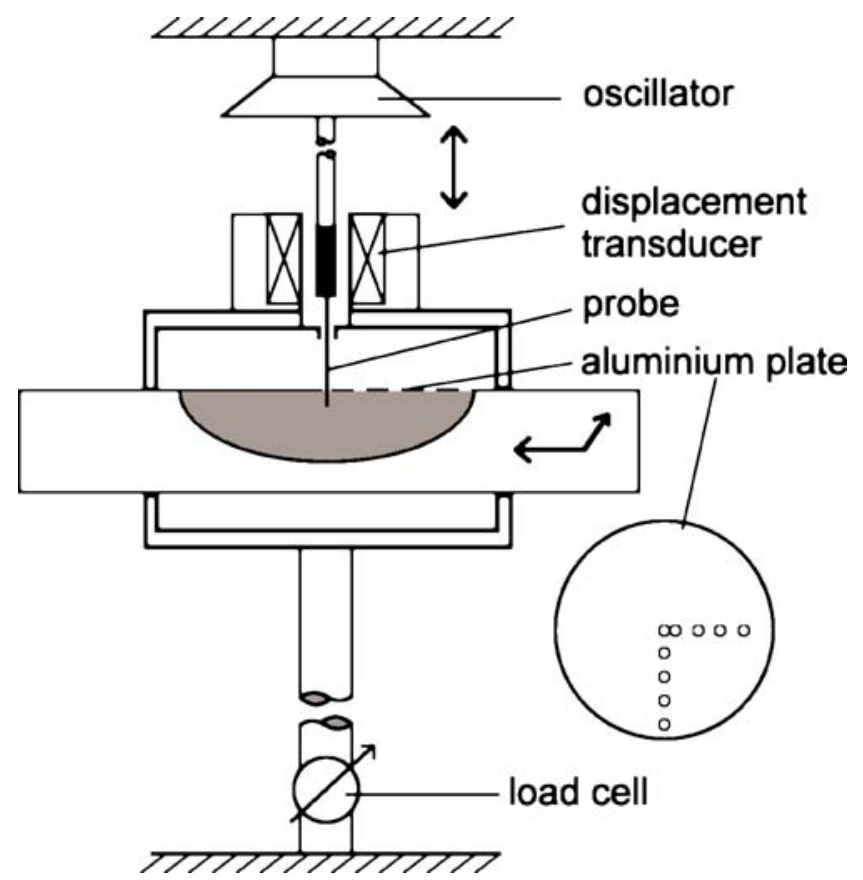

Fig. 1 Schematic drawing of the measurement setup. The top view of the aluminium plate shows the measurement positions. In the horizontal row, the holes are placed at $0,0.5,1.5,2.5$ and $3.5 \mathrm{~mm}$ from the lens centre. In the vertical row, the holes are placed at $0,1.0$, $2.0,3.0$, and $4.0 \mathrm{~mm}$ from the lens centre. The double arrow near the oscillator denotes the direction of the oscillation 
diameter of ca. $0.6 \mathrm{~mm}$ ) at the measuring positions, so that a cylindrical probe could be passed through to the lens at these positions (Fig. 1). The holes were covered with a thin layer of silicone oil (AK1000, Wacker-Chemie $\mathrm{GmbH}$, $\eta=0.97 \mathrm{~Pa} \cdot \mathrm{s})$. The aluminium plate together with the silicone oil prevented the sample from drying.

The cylindrical probe $(0.5 \mathrm{~mm}$ diameter $)$ was lowered until the load cell indicated that the lens surface was reached. The load cell had a resolution of $10^{-6}$ Newton, which was sufficient to measure even the softest lens. In general, the forces were about 10 to 1000 times higher than the resolution of the load cell. The probe was then lowered another $0.5 \mathrm{~mm}$ into the lens. Then, the dynamic measurement was taken over a frequency range of 0.10 to $20 \mathrm{~Hz}$. After the measurement, the probe was raised and moved to the next position. In this way, the lens was scanned, starting in the centre and progressing in $0.5-\mathrm{mm}$ steps up to the periphery, at $4.0 \mathrm{~mm}$ from the centre. A scan was finalized with a repeat measurement in the centre to ensure that the sample had not changed during the measurement (e.g. by drying). The temperature of the sample was registered continuously and was on average $25^{\circ} \mathrm{C}$. Each measurement was carried out with at least two different amplitudes (range 1-50 micrometers) to verify that the material properties were measured in the linear range.

\section{Data analysis}

The raw data consist of the measured reaction forces (amplitude and phase shift) at each frequency and at each position of the probe across the cut lens surface. The measured force, divided by the displacement of the oscillating probe, represents the complex spring constant of the lens. Dividing the spring constant by the shape factor results in the complex shear modulus $\mathrm{G}=\mathrm{G}^{\prime}+\mathrm{i} \cdot \mathrm{G}^{\prime \prime}$. The real part of the complex shear modulus (storage modulus, $\mathrm{G}^{\prime}$ ) represents the elastic component and the imaginary part of the complex shear modulus (loss modulus, $G^{\prime \prime}$ ) represents the energy dissipated or lost as heat [14]. The shape factor is defined by the ratio between the shear modulus of the material and the force applied by the oscillating probe and is derived from the geometry of the sample.

The shape factor was determined by a theoretical analysis, consisting of two steps: (1) by determining an initial shape factor, using a numerical simulation of the test setup and (2) by applying a correction for the nonuniformity of the stiffness.

For the first step of determining the shape factor, a computer model of the test set-up (lens and probe) was created (Fig. 2) using general-purpose finite element software (MSC.Marc 2003, MSC.Software Corporation, Santa Ana, CA, U.S.A.). The required force for a given probe amplitude of oscillation of 10 micrometers was calculated for each position of the probe. The calculated shape factor values (Table 1) were verified by measuring the shear loss modulus of a known material, silicone oil (AK60000, Wacker-Chemie GmbH, $\eta=58.2 \mathrm{~Pa} \cdot \mathrm{s}$ ). The shear loss modulus of this silicone oil is similar to that of a 40-year-old lens. The difference between the measured and calculated shape factor was $1 \%$.

In the second step of determining the shape factor, a correction factor was introduced to account for the

Fig. 2 Finite elements model with the measurement probe in two different positions. The model is shown in the maximum deformed state. For clarity of the figure, the deformations are magnified by a factor of 10
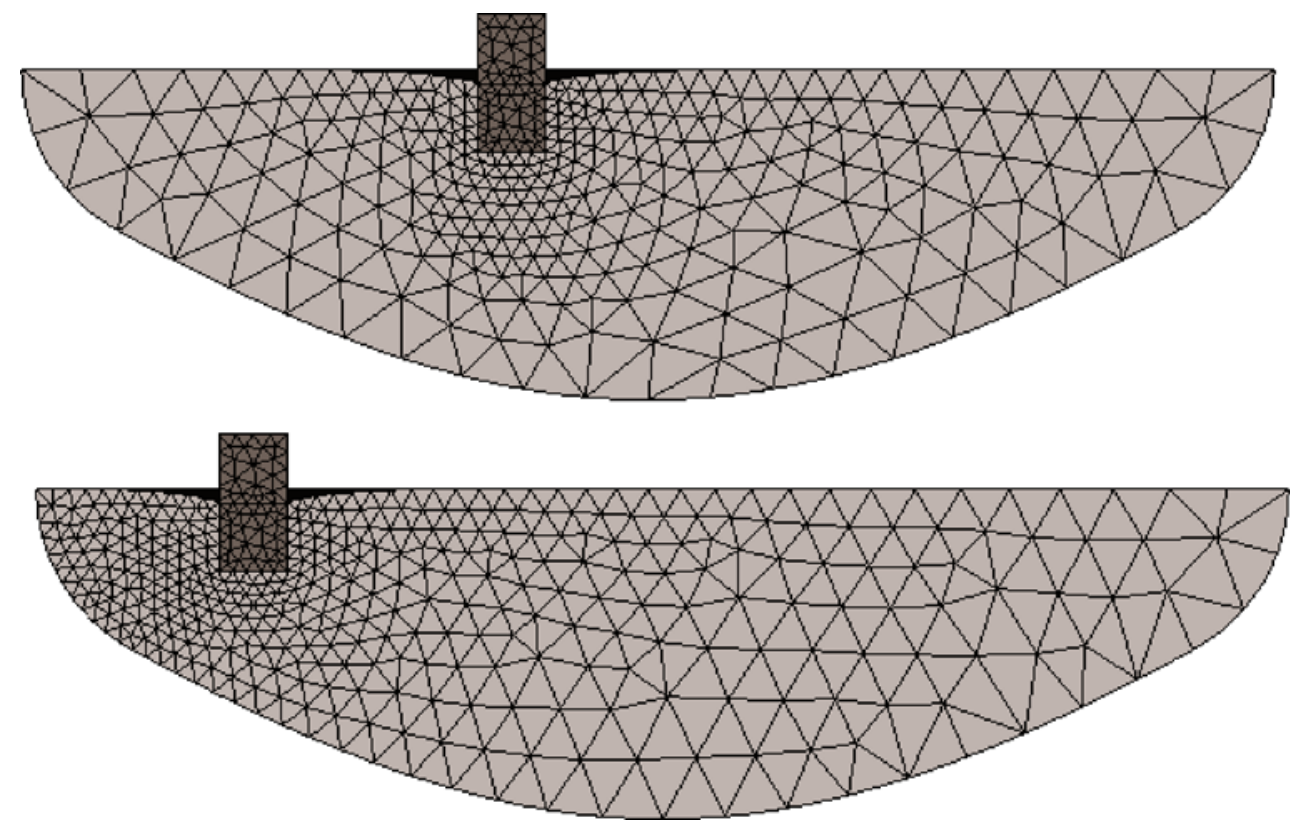
Table 1 Shape factor versus radial position of the probe, determined using the finite elements method

\begin{tabular}{ll}
\hline $\begin{array}{l}\text { Position } \\
{[\mathrm{mm}]}\end{array}$ & $\begin{array}{l}\text { Shape factor } \\
{[\mathrm{mm}]}\end{array}$ \\
\hline 0.0 & 4.36 \\
0.5 & 4.38 \\
1.0 & 4.41 \\
1.5 & 4.47 \\
2.0 & 4.59 \\
2.5 & 4.77 \\
3.0 & 5.09 \\
3.5 & 5.72 \\
4.0 & 7.51 \\
\hline
\end{tabular}

influence of the stiffness gradient on the shape factor. The correction factor was calculated using the average of 'cylindrical shear' ([14], pp 97-98) and 'cylinder compression', which were modified in order to incorporate a nonuniform shear modulus (see Appendix). The correction factor (Table 2) is a function of the measured (uncorrected) ratio between the shear modulus at the lens equator $\left(G_{o}\right)$ and in the lens centre $\left(\mathrm{G}_{\mathrm{i}}\right)$.

Determining the measured shear modulus from the spring constant follows the same two steps: First, the spring constant is divided by the shape factor (Table 1), which results in the (uncorrected) shear modulus and the (uncorrected) ratio $\mathrm{G}_{\mathrm{o}} / \mathrm{G}_{\mathrm{i}}$. Next, the values are corrected by multiplying the (uncorrected) shear modulus with the correction factor in Table 2.

Although the measurements were taken in the frequency range of 0.10 to $20 \mathrm{~Hz}$, only the frequencies $0.10,0.17$ and $0.30 \mathrm{~Hz}$ are reported, since at higher frequencies the mass of the lens (sample inertia) influences the measured force [5].

Table 2 Correction factor for the ratio between the shear modulus at the lens equator $\left(G_{o}\right)$ and in the lens centre $\left(G_{i}\right)$

\begin{tabular}{lll}
\hline True ratio Go/Gi & Uncorrected ratio Go/Gi & Correction factor \\
\hline 0.01 & 0.03 & 0.30 \\
0.1 & 0.21 & 0.47 \\
0.5 & 0.66 & 0.76 \\
1 & 1.00 & 1.00 \\
2 & 1.42 & 1.40 \\
3 & 1.68 & 1.79 \\
4 & 1.83 & 2.18 \\
5 & 1.92 & 2.60 \\
\hline
\end{tabular}

The measured ratio is always closer to 1 than the true ratio, which means that the measurements underestimate the difference between central and equatorial stiffness.

\section{Results}

Repeatability of the measurements was calculated according to the international standard ISO-5725. The repeatability standard deviation, as a percentage of the shear modulus value, was $21 \%$. Figure 3 shows the measured storage and loss shear modulus at 3 frequencies for a 40 -year-old (a) and a 64-year-old (b) lens. Figure 4 shows the storage shear modulus at $0.10 \mathrm{~Hz}$ of 4 lenses ranging in age from 19 to 78 years. For the 10 lenses tested, $\log (\mathrm{G})$ increased with age for all 3 frequencies and at all 9 positions $(\mathrm{P}<0.01$ for all 27 cases). The increase with age was different for the centre and the periphery of the lens: the central storage shear modulus increased by a factor $10^{4}$ and the equatorial storage shear modulus increased by a factor $10^{2}$ over the measured age range.

For all 10 lenses and for all frequencies, the relationship between $\log (\mathrm{G})$ and location on the lens was statistically significant $(\mathrm{P}<0.05)$. The three youngest lenses had a minimum stiffness in the centre of the lens. The peripheral stiffness of these lenses was on average 5.8 times higher than the central stiffness (range 2.2-16.5). The older lenses had a maximum stiffness in the centre of the lens. The centre was on average 64.7 times stiffer than the peripheral stiffness (range 5.8-210).

The two youngest lenses exhibited a maximum stiffness at $2.5-3.0 \mathrm{~mm}$ from the lens centre. The ratio between the storage shear modulus in the centre to storage shear modulus at $\mathrm{R}=3.0 \mathrm{~mm}$ increased exponentially with age ( $\mathrm{P}<0.01$; Fig. 5). The ratio was 0.12 for the 19 -year-old lens and 32 for the 78 -year-old lens.

\section{Discussion}

This study was undertaken to determine the local stiffness of the human crystalline lens. In a previous study, we measured the viscoelastic properties of entire lenses in a large age range [39]. The age correlation observed in the previous study is consistent with the findings in the current study.

\section{Comparisons with existing stiffness data}

Fisher [15] measured the quasi-static Young's modulus of the crystalline lens using a lens-spinning technique. He then calculated separate Young's moduli for nucleus and cortex stiffness to explain the macroscopic anisotropy. This resulted in a cortical stiffness that is higher than the nuclear stiffness for the entire age range of 0-70 years. Fisher's Young's modulus can be compared with our shear modulus results using the equation $\mathrm{G}=1 / 2 \mathrm{E} /(1+\nu)$, with $\mathrm{E}$ being the Young's modulus and $v$ the Poisson's ratio 
Fig. 3 Measured storage $\left(\mathrm{G}^{\prime}\right)$ and loss $\left(\mathrm{G}^{\prime \prime}\right)$ shear modulus at three frequencies, for a 40 - and 64-year-old lens
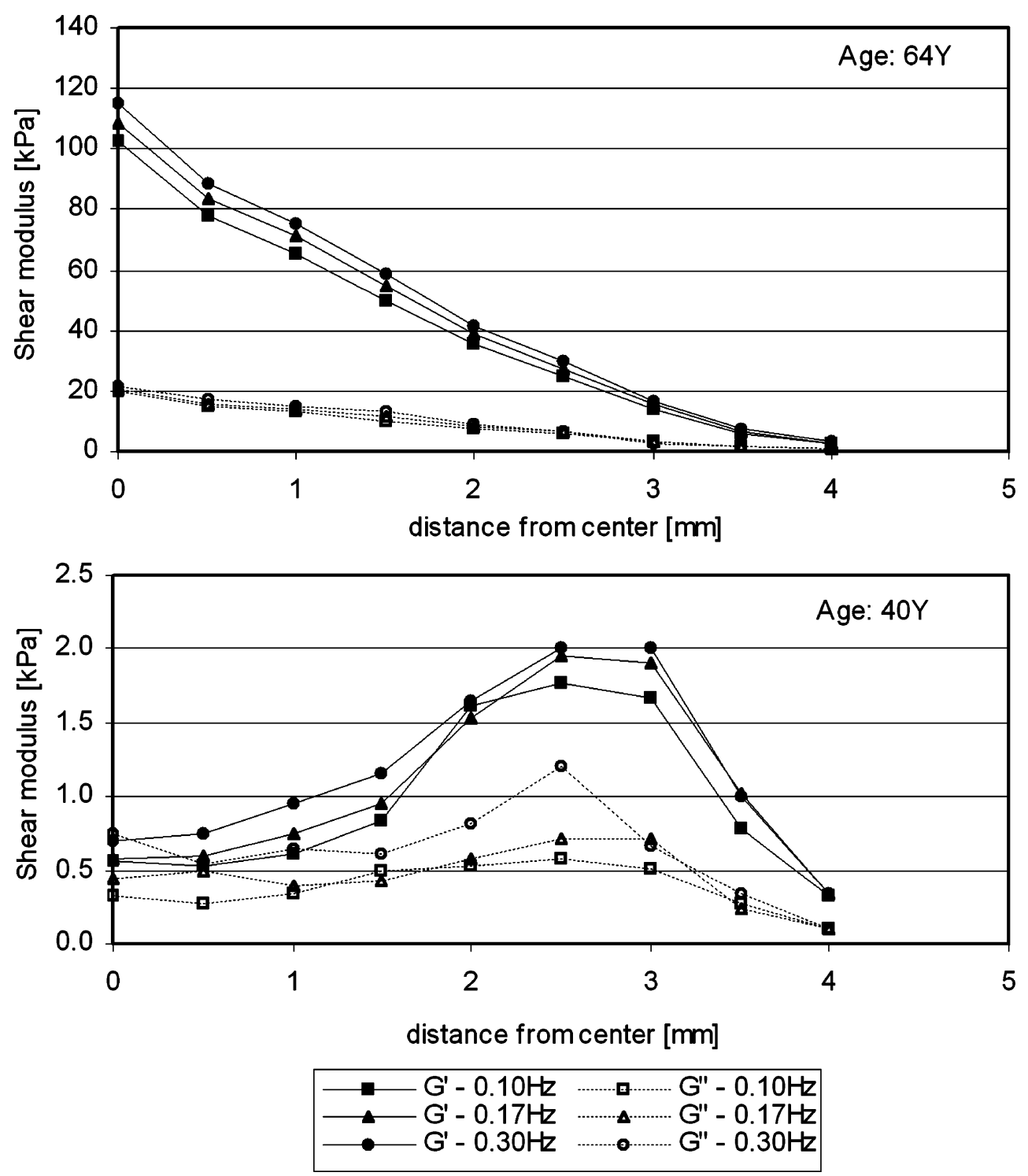

(assumed 0.5). Using this equation, a dramatic difference can be observed between Fisher's results and the results of the current study. We found that at a young age (20 years), the stiffness of the cortex is almost five times higher than the stiffness of the nucleus, while at 70 years of age, the stiffness of the nucleus is 24 times higher than the stiffness of the cortex. At the age of 38 years, at which point Fisher's data shows the largest difference between nucleus and cortex $(1.04 \mathrm{kPa})$, we found a relatively small difference between lens centre and periphery $(0.37 \mathrm{kPa})$. Although our method has some shortcomings (as discussed below), we believe that the current data better reflects the real stiffness gradient of the lens, since it is based on the direct measurement of the stiffness across the cross-section of the lens. Additionally, a recently published analysis suggests that Fisher's conclusion that the cortex is stiffer than the nucleus, particularly in middle age, may be an artefact associated with systematic errors, caused by the modelling assumptions inherent in Fisher's method [9]. Furthermore, the current data seem fairly consistent with published lens hardness data [30].

More recently, Heys et al. [20] used a quasi-static penetration technique to determine local mechanical properties of the lens. In principle, this method determines the local hardness of the lens, but it was converted to shear modulus, assuming a linear relationship between hardness and stiffness. This relationship between hardness and stiffness is not obvious, especially when mechanical properties are non-linear material. Comparing the regression lines between 20 and 70 years of age, we found that the modulus in the centre of the lens increased from $0.023 \mathrm{kPa}$ to $134 \mathrm{kPa}$ (Heys et al.: from $0.040 \mathrm{kPa}$ to $19 \mathrm{kPa}$ ) and the modulus in the periphery of the lens increased from $0.20 \mathrm{kPa}$ to $14 \mathrm{kPa}$ (Heys et al.: $0.089 \mathrm{~Pa}$ to 
Fig. 4 Shear modulus of four lenses across the age range, plotted on a logarithmic scale

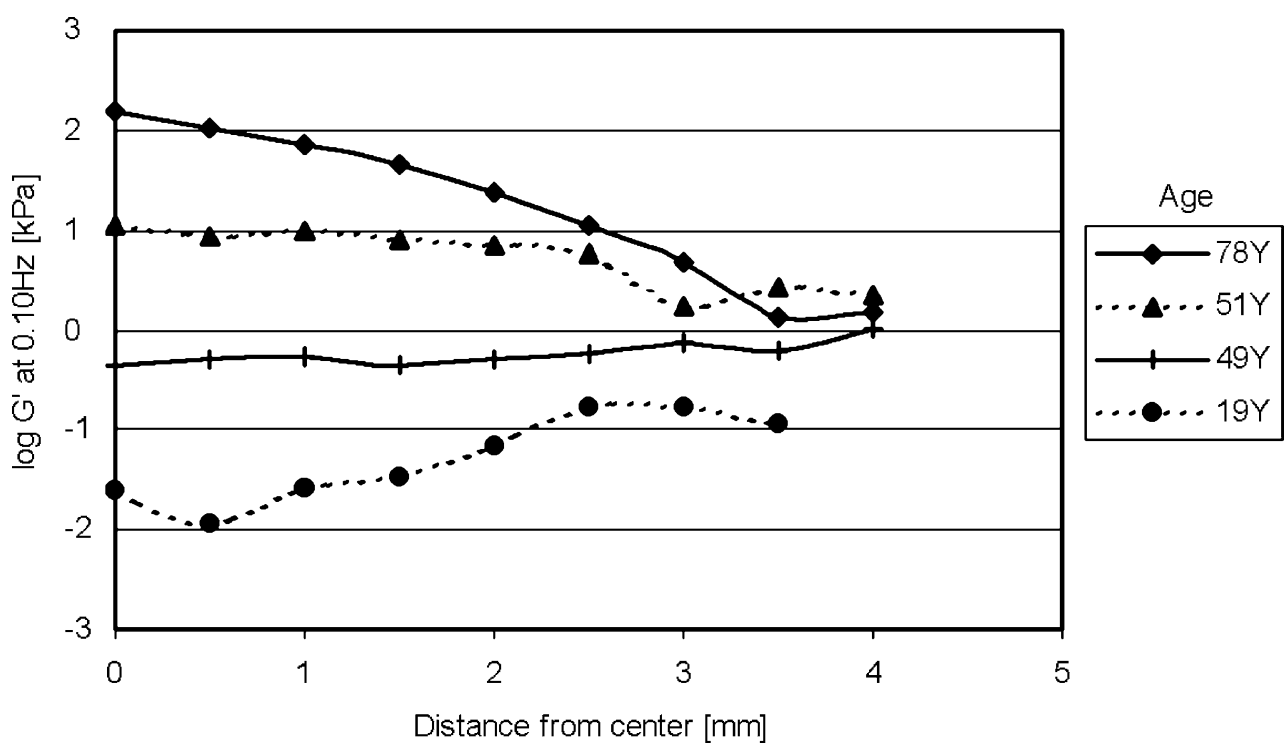

$1.9 \mathrm{kPa}$ ). Despite the differences in methods and assumptions, the trends they found for the relationship between the nuclear and cortical mechanical properties with age support our results nicely.

Limitations of the test method

Measuring the stiffness modulus of the crystalline lens is a difficult task. Generally, the mechanical properties of human tissues are non-linear [17]. In simple terms, this means that the stress-strain curve is not a straight line. This was well-demonstrated for the capsular bag by Krag et al. [27]. Their study also showed a close-to-linear behaviour for small strains. Two other studies also demonstrated this behaviour in the crystalline lens $[16,39]$. In the current study, the measurements were taken in the linear elastic part of the stress-strain curve.

Due to logistic limitations, the lenses used in the current study were stored in a frozen state, prior to the experiment. Therefore, our results only apply to previously frozen lenses. The effect of freezing on the measured shear modulus has been discussed extensively in a previous publication [39] and the arguments also apply to this study's results. In the previous study, pairs of porcine lenses were measured, one fresh and the other after being stored in a frozen state. The difference between fresh and frozen
Fig. 5 Ratio (log-scale) between central and peripheral stiffness versus age, based on the storage shear modulus at $0.1 \mathrm{~Hz}$. The central modulus is taken as the nuclear stiffness and the modulus at $3 \mathrm{~mm}$ away from the centre is taken as the peripheral stiffness

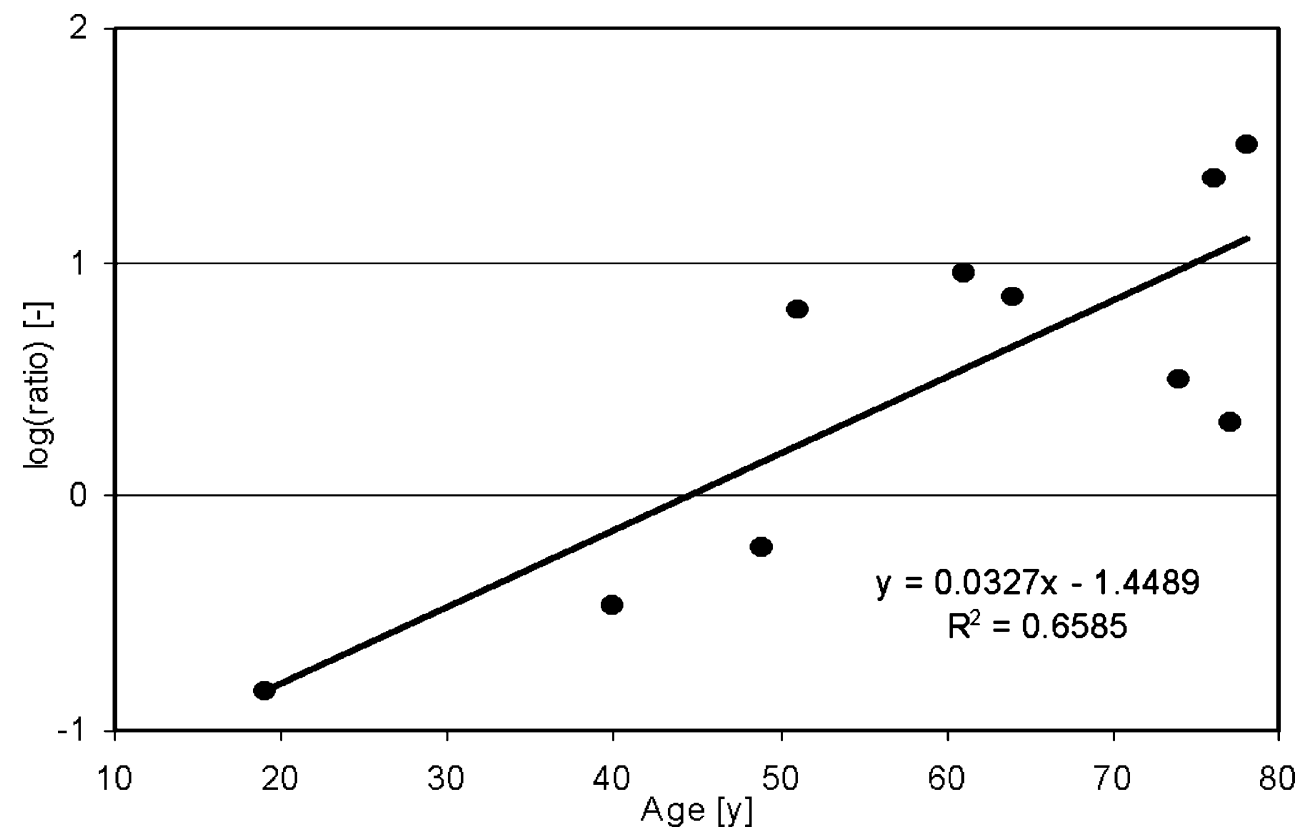


lenses was $8 \%$ in storage compliance and $32 \%$ in loss compliance. This can be considered small when compared to the very large changes of modulus with age and position.

The structure of the lens material directly in contact with the probe will be disturbed. The effect of the disturbance on the measurement result is unknown. A correction factor was calculated during the data analysis, assuming a radially linear stiffness distribution. This does not mean that the measured stiffness distribution is also radially linear. The two younger lenses show a highly non-linear stiffness distribution.

In our study, the analysis has some influence on the observed trends. Figure 6 shows the spring constant $\left(C^{\prime}\right)$ in arbitrary units of two lenses, together with the final storage shear modulus at $0.1 \mathrm{~Hz}$. The figure shows that the conversion to shear modulus has some influence on the shape and the absolute value of the shear modulus, but the relative magnitudes are hardly affected.

Although the fibre cell structure in the crystalline lens suggests anisotropy of the mechanical properties, rabbit lens experiments by Itoi [21] and human lens experiments by Fisher [15] indicate that the anisotropy is relatively small compared to the large differences of shear modulus with position and with age found in the current study.

The number of lenses used in this study was small. Although several prior studies that have considered the effects of age on the lens have used even fewer lenses [6, 7, $25,32]$, the fact that the age related trends are observed despite the small number of lenses used indicates the significance of the age related changes. This study may be confirmed by a larger study, and preferably avoiding freezing the lenses before the measurement.

Lens changes and stiffness profiles

There can be several causes for the increasing stiffness of the lens. Recently, Al-Ghoul et al. [1] quantified the compaction of the lens fibres in the fetal and embryonic nucleus of adult lenses. The authors suggested that lens fibre compaction may cause an increase in lens hardness, which contributes to the loss of accommodation. We found
Fig. 6 Measured spring constant $\left(\mathrm{C}^{\prime}\right)$ in arbitrary units and resulting calculated shear modulus $\left(\mathrm{G}^{\prime}\right)$ for a 40 - and 64-yearold lens

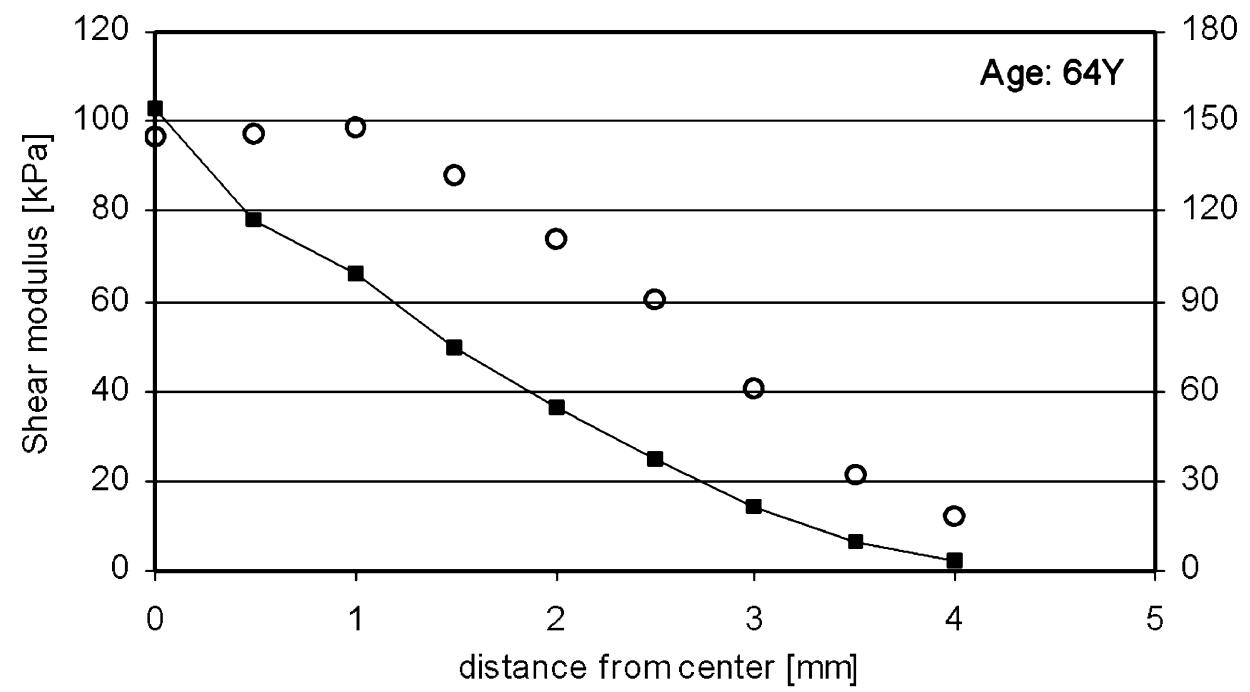

180

150

5
2
$\frac{1}{4}$
$\frac{c}{0}$
0
0
0
0
0
0
0
$\frac{.}{0}$
0
0

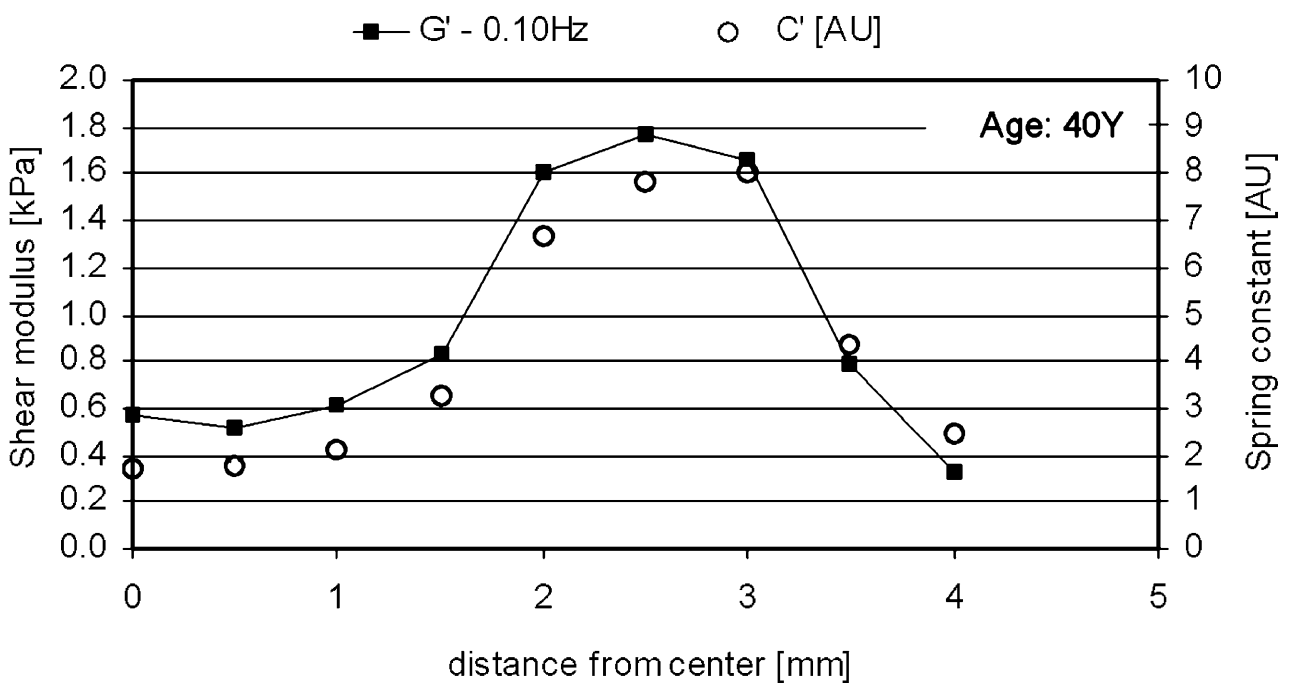


that the change of lens stiffness with age differs between centre and periphery. On a morphological and biochemical level, several age-related changes in the lens occur. The mechanisms by which these age-related changes in the lens occur are different between nucleus and cortex [37]. This may explain, in part, our finding that the rate of lens stiffening with age is different for the centre and the periphery.

A relationship between nuclear hardness and presbyopia was suggested by Pay and Kranz [30]. Here we should note that in ophthalmic literature, there is often a confusion between the terms 'hardness' and 'stiffness,' which is at least partly due to different definitions of hardness [34]. While stiffness defines the relationship between stress and strain, lens hardness is often measured by penetration/ guillotine experiments, so that it depends on the force at which the material breaks. For non-linear mechanical properties in general, the force at break and the stiffness are not proportional.

In the current study, the three youngest lenses of the prepresbyopic age had a soft centre. For lenses of these ages, the difference between centre and periphery was much larger than the suggested differences in measurements of anisotropy $[15,21]$. As an exploratory analysis, the shear modulus was determined as a function of all data, meaning as a function of age and position. A single function was used, $\log (\mathrm{G})=\mathrm{f}($ age, position), being a two variable $3 \mathrm{rd}$ order polynomial, which was fitted through 90 points (10 lenses, so 10 ages, with 9 measured positions per lens), using the least squares method (Fig. 7). No effort was made to determine the goodness of fit and the figure only serves to demonstrate the type of trend that was found in this study. According to this trend analysis, the stiffness of the crystalline lens is generally uniform throughout the entire lens at the age of 45 years (at least in the equatorial plane). This also follows from the ratio between the stiffness of the centre and the stiffness of the periphery (Fig. 5).

\section{Implications}

This study involves a limited number of lenses over the age range of 19 to 78 years old. These measurements showed the following trends: 1) the centre was softer than the periphery in the three youngest lenses; 2) the centre was stiffer than the periphery in older lenses; 3 ) the stiffness of both centre and periphery increase with age, but at a different rate.

The ratio between the stiffness of centre and the stiffness of the periphery could have a special relationship with the onset of presbyopia. If we want to know the influence of the stiffness profile on accommodation and presbyopia, we should determine the influence of the stiffness profile on the pliability of the total lens. This could be done by mechanical calculations. Burd et al. [8] already included a nucleus in their mechanical model, while preliminary investigations, also based on the finite elements method, have demonstrated that the accommodative amplitude is very sensitive to the relative stiffness of the nucleus [38].
Fig. 7 Trend graph of the shear modulus versus position in the lens and age. The line at the bottom is the 20-year-old lens. The modulus increases with age for all positions in the lens. The line at the top is the 70 -year-old lens. Measurements were taken up to $4.0 \mathrm{~mm}$ from the lens centre. The lines are extrapolated to a radius of $4.5 \mathrm{~mm}$ (lens diameter $9.0 \mathrm{~mm}$ )

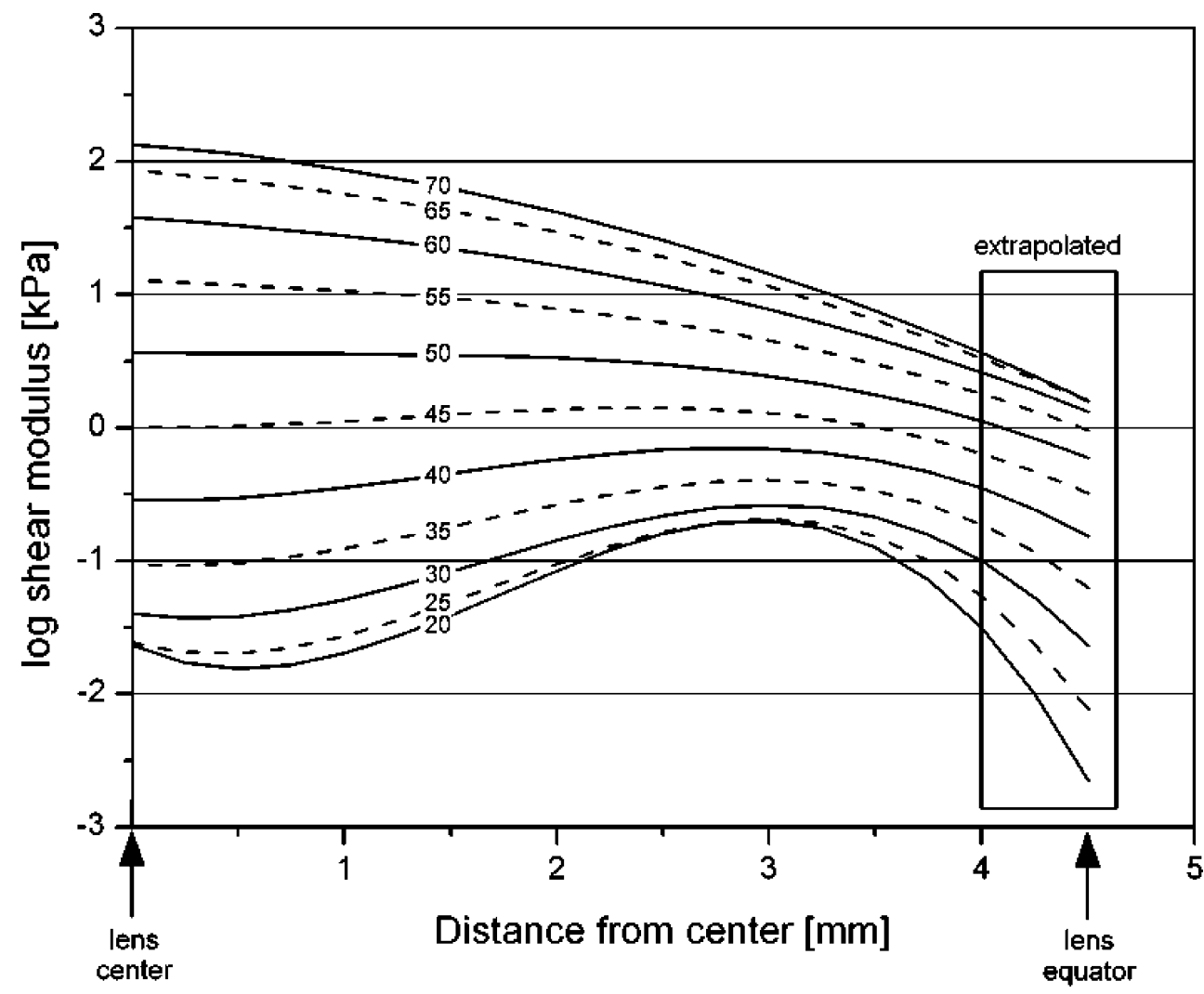


Future research efforts could concentrate on the physiological mechanisms that are responsible for the changes in lens stiffness.

\section{Appendix}

Shape factor and correction for a non-uniform material

Cylindrical shear with a non-uniform modulus:

A probe with radius $r_{i}$ is inserted in a cylinder with radius $r_{o}$. For a uniform material, the shape factor is ([14], pp 98):

$M=\frac{2 \pi \cdot l}{\ln \left(\frac{r_{o}}{r_{i}}\right)}$.

A non-uniform shape-factor is introduced:

$\mathrm{G}^{*}(r)=\mathrm{G}_{\mathrm{c}}^{*}+\mathrm{B}^{*} \mathrm{r}$

with $G_{c}^{*}$ being the central stiffness and $B$ the stiffness slope.

For an infinitesimal element of sample material (width of $\mathrm{dr}$, height of $\mathrm{dw}$ ), the shear stress is:

$\tau=-\mathrm{G}^{*}(\mathrm{r})^{*}(\mathrm{dw} / \mathrm{dr})$.

When $\mathrm{F}$ is the applied force and 1 is the length of the probe, the shear force is also:

$\tau=\mathrm{F} / 2 \pi \mathrm{rl}$

so that:

$(\mathrm{dw} / \mathrm{dr})=-\mathrm{F} /\left\{\mathrm{G}^{*}(\mathrm{r})^{*} 2 \pi \mathrm{rl}\right\}$.

This leads to the integral for the deflection:

$$
\begin{aligned}
\mathrm{w}(\mathrm{r}) & \left.=\int_{\mathrm{r}_{\mathrm{i}}}^{\mathrm{r}} \frac{\partial \mathrm{w}}{\partial \mathrm{r}} \mathrm{dr}=\frac{-\mathrm{F}}{2 \pi \mid \mathrm{G}_{\mathrm{c}}^{*}}\left\{\ln (\mathrm{r})-\ln \left(\mathrm{G}_{\mathrm{c}}^{*}+\mathrm{B}^{*} \mathrm{r}\right)\right\}\right]_{\mathrm{r}_{\mathrm{i}}}^{\mathrm{r}}= \\
& =\frac{-\mathrm{F}}{2 \pi l \mathrm{G}_{\mathrm{c}}^{*}}\left\{\ln \left(\frac{\mathrm{r}}{\mathrm{r}_{\mathrm{i}}}\right)-\ln \left(\frac{\mathrm{G}_{\mathrm{c}}^{*}+\mathrm{B}^{*} \mathrm{r}}{\mathrm{G}_{\mathrm{c}}^{*}+\mathrm{B}^{*} \mathrm{r}_{i}}\right)\right\}+\mathrm{w}\left(\mathrm{r}_{\mathrm{i}}\right)
\end{aligned}
$$

(Handbook of Chem \& Phys. 77th ed, Page A-26, equation 110)

$$
\begin{aligned}
& w\left(r_{o}\right)=0=\frac{-F}{2 \pi l G_{c}^{*}}\left\{\ln \left(\frac{r_{o}}{r_{i}}\right)-\ln \left(\frac{G_{c}^{*}+B^{*} r_{o}}{G_{c}^{*}+B^{*} r_{i}}\right)\right\} \\
& +w\left(r_{i}\right) \\
& w(r)=\frac{+F}{2 \pi l G_{c}^{*}}\left\{\ln \left(\frac{r_{o}}{r}\right)-\ln \left(\frac{G_{c}^{*}+B^{*} r_{o}}{G_{c}^{*}+B^{*} r}\right)\right\} \\
& \frac{1}{C}=\frac{w\left(r_{i}\right)}{P}=\frac{J^{*}}{2 \pi l}\left\{\ln \left(\frac{r_{o}}{r_{i}}\right)-\ln \left(\frac{G_{c}^{*}+B^{*} r_{o}}{G_{c}^{*}+B^{*} r_{i}}\right)\right\}
\end{aligned}
$$

So for the compliance $(\mathrm{J})$ and with:

$\mathrm{G}_{\mathrm{o}}^{*}=$ modulus at $\mathrm{r}=\mathrm{r}_{\mathrm{o}}$

$\mathrm{G}_{\mathrm{i}}^{*}=$ modulus at $\mathrm{r}=\mathrm{r}_{\mathrm{i}}$

$\mathrm{J}^{*}=\frac{1}{\mathrm{C}} * \frac{2 \pi \mathrm{l}}{\ln \left(\frac{\mathrm{r}_{\mathrm{o}}}{\mathrm{r}_{\mathrm{i}}}\right)-\ln \left(\frac{\mathrm{G}_{\mathrm{o}}^{*}}{\mathrm{G}_{\mathrm{i}}^{*}}\right)}$

and for the shear modulus:

$\mathrm{G}_{0}^{*}=\frac{\mathrm{C}}{2 \pi \mathrm{l}}\left\{\ln \left(\frac{\mathrm{r}_{\mathrm{o}}}{\mathrm{r}_{\mathrm{i}}}\right)-\ln \left(\frac{\mathrm{G}_{\mathrm{o}}^{*}}{\mathrm{G}_{\mathrm{i}}^{*}}\right)\right\}$

$\mathrm{M}_{\mathrm{cs}}=2 \pi l /\left\{\ln \left(\frac{r_{o}}{r_{i}}\right)-\ln \left(\frac{G_{o}^{*}}{G_{i}^{*}}\right)\right\}$.

\section{Compression of a cylinder:}

The area directly under the probe is represented by the compression of a cylinder, the cylinder having a linear changing stiffness.

$\sigma=\mathrm{E} \varepsilon$

and for a material with Poisson's ratio of 0.5 :

$\varepsilon=\sigma / \mathrm{E}=\sigma / 3 \mathrm{G}$

with $\sigma$ the normal stress, E the Young's modulus and $\varepsilon$ the relative compression ( $\mathrm{dl} / \mathrm{l}$ for a uniform stiffness). Each slice $(\mathrm{dz})$ at vertical position $\mathrm{z}$ below the probe is compressed, influenced by the local shear modulus $\mathrm{G}(\mathrm{z})$ :

$\frac{\mathrm{d}(\mathrm{dz})}{\mathrm{dz}}=\sigma / 3 \mathrm{G}(\mathrm{z})=\mathrm{F} / 3 \pi \mathrm{r}_{\mathrm{i}}^{2} \mathrm{G}(\mathrm{z})$.

The total compression of the cylinder:

$z=\int_{0}^{h} \frac{d(d z)}{d z} d z=\int_{0}^{h} \frac{F}{3 \pi r_{i}^{2} G(z)} d z$.

Introducing a non-uniform material:

$\mathrm{G}(\mathrm{z})=\mathrm{G}_{\mathrm{o}}\left(1+\mathrm{D}^{*} \mathrm{z}\right)$

With Eq. (15), Eq. (14) becomes:

$z=\frac{F}{3 \pi R^{2} G_{o}} \int_{0}^{h} \frac{1}{1+D \cdot z} d z=\frac{F \cdot \ln (1+h \cdot D)}{3 \pi r_{i}^{2} G_{o} D}$.

And for the shape factor:

$M_{c c}=\frac{F / z}{G_{o}}=\frac{3 \pi r_{i}^{2} \cdot D}{\ln (1+h \cdot D)}$.

In the data analysis, a cylinder height of $2.0 \mathrm{~mm}$ was used. 
The average correction factor is defined by:

$0.5^{*}\left\{\frac{M_{c s}\left(G_{o} / G_{i}\right)}{M_{c s}\left(G_{o} / G_{i}=1\right)}+\frac{M_{c c}\left(G_{o} / G_{i}\right)}{M_{c c}\left(G_{o} / G_{i}=1\right)}\right\}$.

\section{References}

1. Al-Ghoul KJ, Nordgren RK, Kuszak AJ, Freel CD, Costello MJ, Kuszak JR (2001) Structural evidence of human nuclear fiber compaction as a function of ageing and cataractogenesis. Exp Eye Res 72(3):199-214

2. van Alphen GW, Graebel WP (1991) Elasticity of tissues involved in accommodation. Vis Res 31:1417-1438

3. Atchison DA (1995) Accommodation and presbyopia. Ophthalmic Physiol Opt 15(4):255-272

4. Beers AP, van der Heijde GL (1994) In vivo determination of the biomechanical properties of the component elements of the accommodation mechanism. Vision Res 34(21):2897-2905

5. Birnboim MH, Ferry JD (1961) Method for measuring dynamic mechanical properties of viscoelastic liquids and gels; the gelation of polyvinyl chloride. J Appl Physi 32(11):2305-2313

6. Boettner EA, Wolter JR (1962) Transmission of the ocular media. Invest Ophthal 1:776-783

7. Brown N (1973) The change in shape and internal form of the lens of the eye on accommodation. Exp Eye Res 15:441-459

8. Burd H, Judge S, Cross J (2002) Numerical modelling of the accommodating lens. Vision Res 42(18):2235-2251

9. Burd HJ, Wilde GS, Judge SJ (2006) Can reliable values of Young's modulus be deduced from Fisher's (1971) spinning lens measurements? Vision Res (8-9):1346-1360

10. Czygan C, Hartung C (1996) Mechanical testing of isolated senile human eye lens nuclei. Med Eng Phys 18(5):345-349

11. Dubbelman M, Van der Heijde GL, Weeber HA, Vrensen GF (2003) Changes in the internal structure of the human crystalline lens with age and accommodation. Vision Res 43(22):2363-2375

12. Ejiri M, Thompson HE, O’Neill WD (1969) Dynamic viscoelastic properties of the lens. Vision Res 9:233-244

13. Fagerholm PP, Philipson BT, Lindstrom B (1981) Normal human lens - the distribution of protein. Exp Eye Res 33(6):615-620

14. Ferry JD (1980) Viscoelastic properties of polymers. Wiley, New York, pp 672

15. Fisher RF (1971) The elastic constants of the human lens. J Physiol 212:147-180

16. Fukuda M (1962) [Rheological characteristics of human chrystalline lens]. J Jap Ophthalmol Soc 66(11):229(1265)-1241(1277)

17. Fung YC (1993) Biomechanics: mechanical properties of living tissues. Springer, Berlin Heidelberg New York, pp 568

18. Glasser A, Campbell MC (1998) Presbyopia and the optical changes in the human crystalline lens with age. Vision Res 38(2): 209-229

19. Glasser A, Campbell MCW (1999) Biometric, optical and physical changes in the isolated human crystalline lens with age in relation to presbyopia. Vis Res 39(11):1991-2015
20. Heys KR, Cram SL, Truscott RJ (2004) Massive increase in the stiffness of the human lens nucleus with age: the basis for presbyopia? Mol Vis 10:956-963

21. Itoi M, Ito N, Kaneko H (1965) Visco-elastic properties of the lens. Exp Eye Res 4(3):168-173

22. Kikkawa Y, Sato T (1963) Elastic properties of the lens. Exp Eye Res 2:210-215

23. Koretz JF, Cook CA, Kaufman PL (1997) Accommodation and presbyopia in the human eye. Changes in the anterior segment and crystalline lens with focus. Invest-Ophthalmol-Vis-Sci 38(3):569-578

24. Koretz JF, Handelman GH (1982) Model of the accommodative mechanism in the human eye. Vis Res 22:917-927

25. Koretz JF, Handelman GH, Brown NP (1984) Analysis of human crystalline lens curvature as a function of accommodative state and age. Vision Res 24(10):1141-1151

26. de Korte CL, van der Steen AF, Thijssen JM, Duindam JJ, Otto C, Puppels GJ (1994) Relation between local acoustic parameters and protein distribution in human and porcine eye lenses. Exp Eye Res 59(5):617-627

27. Krag S, Olsen T, Andreassen TT (1997) Biomechanical characteristics of the human anterior lens capsule in relation to age. Investig Ophthalmol Vis Sci 38(2):357-363

28. Kuwahara K (1980) [Visco-elasticity of bovine lens]. Nippon Ganka Gakkai Zasshi 84(9):1360-1366

29. Patnaik B (1967) A photographic study of accommodative mechanisms: changes in the lens nucleus during accommodation. Inv Ophthalmol 6:601-611

30. Pau H, Kranz J (1991) The increasing sclerosis of the human lens with age and its relevance to accommodation and presbyopia. Graefe's Arch Clin Exp Ophthalmol 299:294-296

31. Pierscionek BK (1993) In vitro alteration of human lens curvatures by radial stretching. Exp Eye Res 57(5):629-635

32. Pierscionek BK (1995) Age-related response of human lenses to stretching forces. Exp Eye Res 60(3):325-332

33. Pierscionek BK, Augusteyn RC (1991) Structure/function relationship between optics and biochemistry of the lens. Lens Eye Toxic Res 8(2-3):229-243

34. Smith JM, El-Brawany M, Nassiri D, Tabandeh H, Thompson GM (2002) The relationship between nuclear colour and opalescence on the LOCSIII scale and physical characteristics of cataract nuclei. Eye 16(5):543-551

35. Tabandeh H, Thompson GM, Heyworth P, Dorey S, Woods AJ, Lynch D (1994) Water content, lens hardness and cataract appearance. Eye 8(Pt 1):125-129

36. Taylor VL, al-Ghaul KJ, Lane CW, Davis VA, Kuszak JR, Costello MJ (1996) Morphology of the normal human lens. Invest Ophthalmol Vis Sci 37(7):1396-1410

37. Vrensen GF (1995) Aging of the human eye lens-a morphological point of view. Comp Biochem Physiol A Physiol 111(4):519-532

38. Weeber HA (1999) A finite elements model of accommodation; first results. Vision science and its applications (annual meeting): OSA technical digest. Optical Society of America, Washington DC, pp 114-116

39. Weeber HA, Eckert G, Soergel F, Meyer CH, Pechhold W, van der Heijde RGL (2005) Dynamic mechanical properties of human lenses. Exp Eye Res 80(3):425-434 